OPEN ACCESS

Edited by:

S. Lee Hong,

Ohio University, USA

Reviewed by:

Breanna Erin Studenka,

Utah State University, USA

Kristina Anne Neely,

The Pennsylvania State University,

USA

Leslie Marion Decker,

Université de Caen

Basse-Normandie, France

*Correspondence:

Christoph Maurer,

Klinik für Neurologie und

Neurophysiologie, Universität Freiburg, Breisacherstr. 64, Freiburg 79106, Germany christoph.maurer@ uniklinik-freiburg.de

Received: 18 February 2015 Accepted: 07 May 2015

Published: 23 June 2015

Citation:

Wiesmeier IK, Dalin D and Maurer C

(2015) Elderly use proprioception rather than visual and vestibular cues for postural motor control.

Front. Aging Neurosci. 7:97. doi: 10.3389/fnagi.2015.00097

\section{Elderly use proprioception rather than visual and vestibular cues for postural motor control}

\author{
Isabella Katharina Wiesmeier, Daniela Dalin and Christoph Maurer* \\ Klinik für Neurologie und Neurophysiologie, Universität Freiburg, Freiburg, Germany
}

Multiple factors have been proposed to contribute to the deficits of postural control in the elderly. They were summarized as sensory, motor, and higher-level adaptation deficits. Using a model-based approach, we aimed to identify which of these deficits mainly determine age-related changes in postural control. We analyzed postural control of 20 healthy elderly people with a mean age of 74 years. The findings were compared to data from 19 healthy young volunteers (mean age 28 years) and 16 healthy middleaged volunteers (mean age 48 years). Postural control was characterized by spontaneous sway measures and measures of perturbed stance. Perturbations were induced by pseudorandom anterior-posterior tilts of the body support surface. We found that spontaneous sway amplitude and velocity were significantly larger, and sway frequencies were higher in elderly compared to young people. Body excursions as a function of tilt stimuli were clearly different in elderly compared to young people. Based on simple feedback model simulations, we found that elderly favor proprioceptive over visual and vestibular cues, other than younger subjects do. Moreover, we identified an increase in overall time delay challenging the feedback systems stability, and a decline in the amplitude of the motor feedback, probably representing weakness of the motor system. In general, these parameter differences between young and old may result from both deficits and compensation strategies in the elderly. Our model-based findings correlate well with deficits measured with clinical balance scores, which are widely used in clinical practice.

Keywords: elderly, postural control, proprioception, vestibular, model

\section{Introduction}

Postural control in elderly people is impaired by numerous factors [for an overview, see, e.g., Shumway-Cook and Woollacott (2001) and Iosa et al. (2014)]. Changes in sensory systems include a reduced joint position sense at the ankle (Horak et al., 1989; Goble et al., 2009), paralleled by a higher perception threshold for vibration (Tang and Woollacott, 1996; Hilz et al., 1998; Lin et al., 2005; Shaffer and Harrison, 2007). Moreover, visual function (visual acuity, contour and depth perception, contrast sensitivity, peripheral vision) is reduced, partly due to structural changes of the eye. In addition, a decrease of vestibular function has been described (Bergström, 1973; Rosenhall, 1973; Merchant et al., 2000; Park et al., 2001; Rauch et al., 2001; Shumway-Cook and Woollacott, 2001; Nag and Wadhwa, 2012; Grossniklaus et al., 2013). 
Impairments of the motor system in the elderly have numerously been reported [e.g., Doherty (2003), Macaluso and De Vito (2004), and Reeves et al. (2006)]. For example, a 40\% reduction of the lower body muscle strength was found when compared to young healthy adults (Shumway-Cook and Woollacott, 2001). During balance corrections, elderly people display an altered muscle response organization (Shumway-Cook and Woollacott, 2001; Tsai et al., 2014) and more frequent coactivations of antagonist muscles (Shumway-Cook and Woollacott, 2001; Macaluso and De Vito, 2004; Klass et al., 2007; Papegaaij et al., 2014).

Some authors proposed deficits in higher-level adaptive systems [e.g., Shumway-Cook and Woollacott (2001)]. They suggested that elderly people's ability to adapt to external perturbations is diminished (Horak et al., 1989; Peterka and Black, 1990; Mansfield and Maki, 2009). Elderly people react with longer onset latencies to external perturbations than young adults do (Woollacott et al., 1988; Horak et al., 1989; Woollacott and ShumwayCook, 1990; Tsai et al., 2014). In addition, elderly people seem to have difficulties in sensory reweighting (Horak et al., 1989; Teasdale and Simoneau, 2001; Eikema et al., 2012, 2014). The term "sensory reweighting" was established by Nashner et al. (1982) to describe a process of scaling the relative importance of sensory cues (visual, vestibular, and proprioceptive) for motor control (Nashner et al., 1982; Jeka et al., 2006). However, the sensory weighting process itself seems to be unimpaired in elderly people (Allison et al., 2006; Jeka et al., 2006), indicating that differences in sensory weights between elderly and young people are related to different sensory preferences.

Measures of human postural control are usually segregated into spontaneous sway measures and measures of motor behavior induced by external perturbations. Age-related differences in spontaneous sway mainly concern increases in mean velocity (MV) and mean frequency (MF) (Maki et al., 1990; Hytönen et al., 1993; Baloh et al., 1994; Collins et al., 1995; Prieto et al., 1996; Maurer and Peterka, 2005; Qu et al., 2009).

During perturbed stance, somatosensory cues affect postural control in young people differently than in elderly people [e.g., Peterka and Black (1990), Speers et al. (2002), Fransson et al. (2004), Ghulyan et al. (2005), and Maitre et al. (2013)]. In general, stance of elderly people is reported to be less stable with absent or altered proprioceptive, vestibular, and visual information (Peterka and Black, 1990; Whipple et al., 1993; Speers et al., 2002; Rosengren et al., 2007; Liaw et al., 2009; Pierchała et al., 2012; Maitre et al., 2013; Eikema et al., 2014), leading to larger body excursions. Some authors evaluated the relationship between the perturbation and the induced body motion in terms of transfer functions [see Materials and Methods, see also Nashner and McCollum (1985), Ishida et al. (1997), Van der Kooij et al. (2001), Peterka (2002), Maurer and Peterka (2005), Masani et al. (2006), Maurer et al. (2006a), Vette et al. (2010), Davidson et al. (2011), Nishihori et al. (2012), and Van der Kooij and Peterka (2011)]. Transfer functions are frequently interpreted using a model-based approach. These models usually involve inverted pendulum bodies, a neural controller including a proportional (stiffness of the system) and a derivative feedback gain (damping of the system), and a feedback time delay. The proportional gain is proportional to the sensory error signal and the derivative gain is proportional to the time derivative of the sensory error signal. Both factors are added up as a motor output to provide corrective ankle torque, thereby stabilizing the inverted pendulum and reducing oscillations (Peterka, 2002; Vette et al., 2010). The feedback time delay represents the lumped time delays of sensory, central, and motor transduction (Peterka, 2002). The sensory weighting mechanism of the model scales the gains of the sensory cues (proprioceptive, vestibular, and visual) in terms of relative contributions to the overall feedback gain (Maurer et al., 2006a; Van der Kooij and Peterka, 2011; Engelhart et al., 2014).

Recently, some authors applied the model-based approach to postural control data of elderly people (Cenciarini et al., 2009, 2010; Davidson et al., 2011; Nishihori et al., 2012; Maurer and Peterka, 2005). During quiet stance, elderly people seem to have an increased proportional gain of the sensorimotor control system (Maurer and Peterka, 2005; Nishihori et al., 2012). During external perturbations, an increased derivative gain of the system has been reported (Cenciarini et al., 2009, 2010; Davidson et al., 2011), whereas reports of the system's proportional gain were controversial (Cenciarini et al., 2009, 2010; Davidson et al., 2011), depending, for example, on the direction of sway.

In the current study, we aimed to find out whether we are able to detect sensory, motor, and higher-level adaptation deficits mentioned above in postural control behavior of the elderly. In addition, we aimed to identify which of these contributors most significantly influence postural control in the elderly. For that, we assessed both, spontaneous sway parameters and applied external perturbations in young, middle-aged, and elderly people. The subjects' reactions to anterior-posterior platform tilts were analyzed at 11 frequencies with eyes closed or open, using different amplitudes, with the purpose to simultaneously identify the major components of the sensorimotor control system and their modifications as a function of age. We hypothesized that degradations of sensory, motor, and higher-level adaptation deficits in elderly could be extracted from postural control behavior. As our modelbased approach is highly sensitive to changes in the sensory-motor system, it might be valuable in future for differentiating between age-related and pathology-related impairments of postural control, and for evaluating therapeutic interventions that ameliorate postural control in elderly.

\section{Materials and Methods}

Subjects were tested by recording spontaneous sway as well as motor reactions to platform tilts (perturbed stance). In addition, elderly subjects were tested using relevant standardized clinical tests.

\section{Subjects}

In this study, we measured postural control of elderly people (60-80 years group) and compared their data with data from middle-aged (40-59 years group) and young (20-39 years group) people.

The group of elderly subjects consisted of 20 participants with a mean age of $74 \pm 3.4$ years (Mean $\pm S D, 10$ female, 10 male). We excluded elderly people suffering from any disease that may interact with postural control. For that, each subject was carefully 
examined by a senior consultant neurologist. In addition, we specifically tested for vestibular function using Frenzel goggles on a turning chair. We thereby quantified the function of the vestibular-ocular reflex. Moreover, we evaluated proprioceptive function, which is related to deep sensibility, by testing for position sense and by quantifying vibration sense using a vibrating tuning fork. Further exclusion criteria included any acute or chronic disease that may influence the general condition of health.

Elderly subjects were also assessed using the timed up and go test (TUG, Enkelaar et al., 2013) and the functional reach test (FRT, Enkelaar et al., 2013). Our emphasis was to monitor elderly people's balance function with widely accepted clinical tests. Furthermore, we correlated those relevant clinical tests with our postural control measurements to evaluate whether functional impairments shown by one of our parameters may be linked to abnormalities in clinical tests.

For comparison, we analyzed data of 16 healthy volunteers (middle-aged group) between 36 and 58 years with a mean age of $48.2 \pm 5.3$ years ( 9 female, 7 male) who had been measured in our laboratory during the previous years. In addition, data of 19 healthy volunteers (20-39 years group) between 22 and 34 years with a mean age of $27.6 \pm 3.7$ years (10 female, 9 male) were acquired.

\section{Procedures}

Spontaneous sway and perturbed stance were measured on a custom-built motion platform (Cnyrim et al., 2009; Figure 1). For that, participants were told to stand upright in a relaxed position on the platform, wearing their normal shoes. Stance width was predetermined within a marked area (maximum $30 \mathrm{~cm}$ ). For safety reasons, participants held two ropes hanging from the ceiling in a way they were not able to attain any orientation cues (Cnyrim et al., 2009). However, no subject fell, probably due to the small stimulus amplitudes. Spontaneous sway was measured on the non-moving platform with eyes open and with eyes closed. Each trial lasted 1 min. Between each trial, a short break was taken according to the participants needs.

We measured center-of-pressure (COP) sway paths and 3-D angular positions of the body in order to calculate spontaneous sway measures (parameters) and measures of perturbed stance. The COP sway path was detected with the help of a force transducing platform (Kistler platform type 9286, Winterthur, Switzerland, Figure 1). 3-D angular positions (Angular excursions) of the body (hip-to-ankle, shoulder-to-hip) and the platform in space were measured using an optoelectronic motion-measuring device with markers attached to shoulder, hip, and a rigid bar solely fixed to the platform (Optotrak 3020, Waterloo, ON, Canada). Each marker consisted of three light-emitting diodes fixed to a rigid triangle. 3-D angular positions of the triangles were used to calculate marker positions (Maurer et al., 2006b). Optotrak ${ }^{\circledR}$ and Kistler ${ }^{\circledR}$ output signals as well as the stimulus signals were transferred online to a computer system (IBM compatible Pentium ${ }^{\circledR}$ ) via an analog-digital converter at a sampling rate of $100 \mathrm{~Hz}$. We recorded all data with software programed in LabView ${ }^{\circledR}$ (National Instruments, Austin, TX, USA). Center of mass (COM) height above the ankle joints was calculated according to tables from Winter

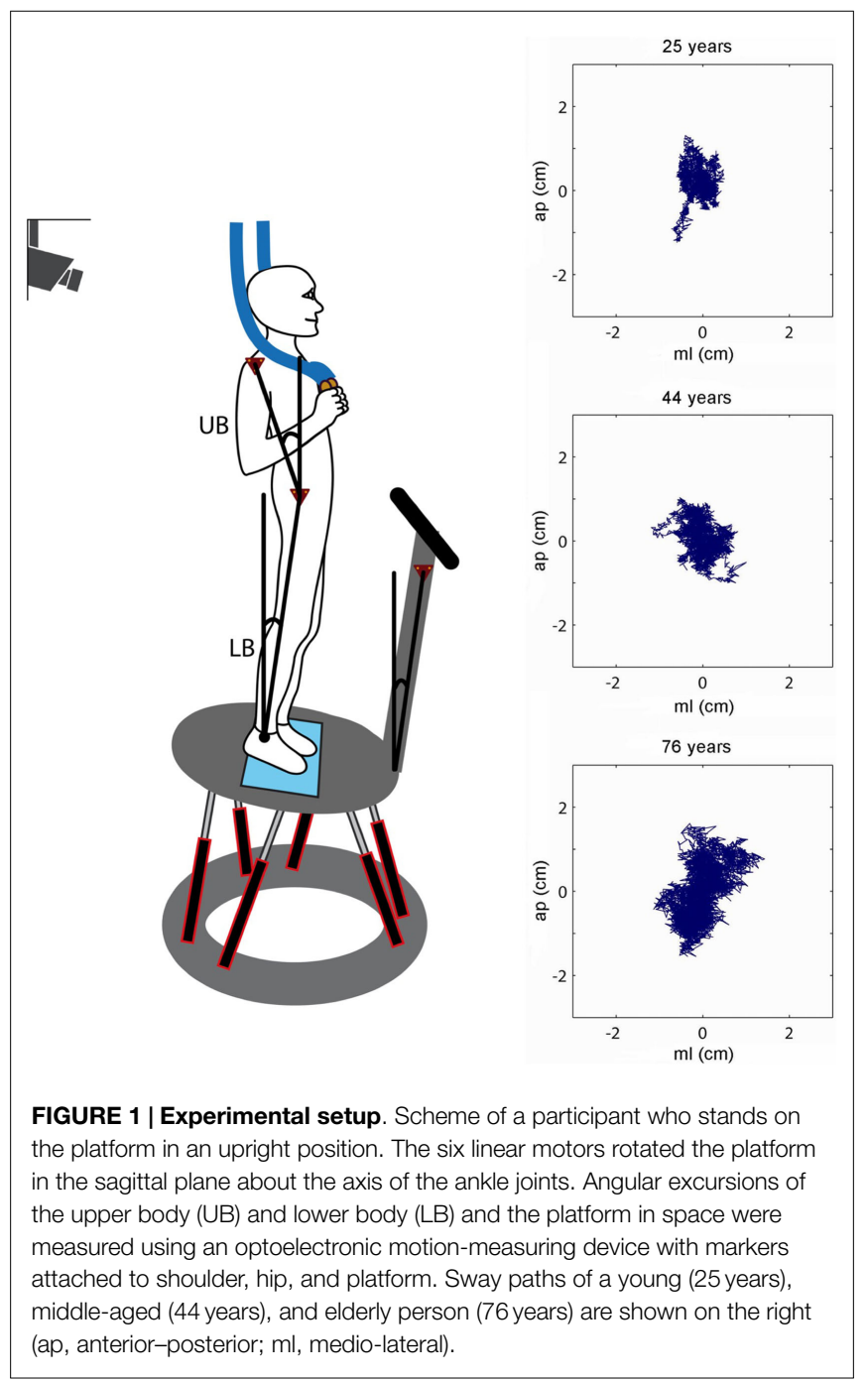

(1995) using the measured heights of hip and shoulder markers. A detailed description of the experimental setup has been published previously (Maurer et al. 2003, 2006a, 2006b; Cnyrim et al., 2009).

Perturbed stance was measured on the moving platform with eyes open and with eyes closed (Figure 2). The rotational tilt is characterized by a platform rotation in the sagittal plane with the tilt axis passing through the participant's ankle joints (Maurer et al., 2006a,b; Cnyrim et al., 2009). Platform rotations were designed as pseudorandom stimuli (PRTS, pseudorandom ternary sequence) with 2 peak angular displacements $\left(0.5^{\circ}\right.$ and $\left.1^{\circ}\right)$ at 11 frequencies $(0.05,0.15,0.3,0.4,0.55,0.7,0.9,1.1,1.35,1.75$, and $2.2 \mathrm{~Hz}$ ).

\section{Data Analysis}

Data analysis was performed off-line with custom-made software programed in MATLAB $^{\circledR}$ (The MathWorks Inc., Natick, MA, USA; Maurer et al., 2003, 2006b; Cnyrim et al., 2009). From the lower and upper body excursions and COP over time in anterior-posterior (and medio-lateral directions), we calculated root mean square (RMS) around the mean COP position 


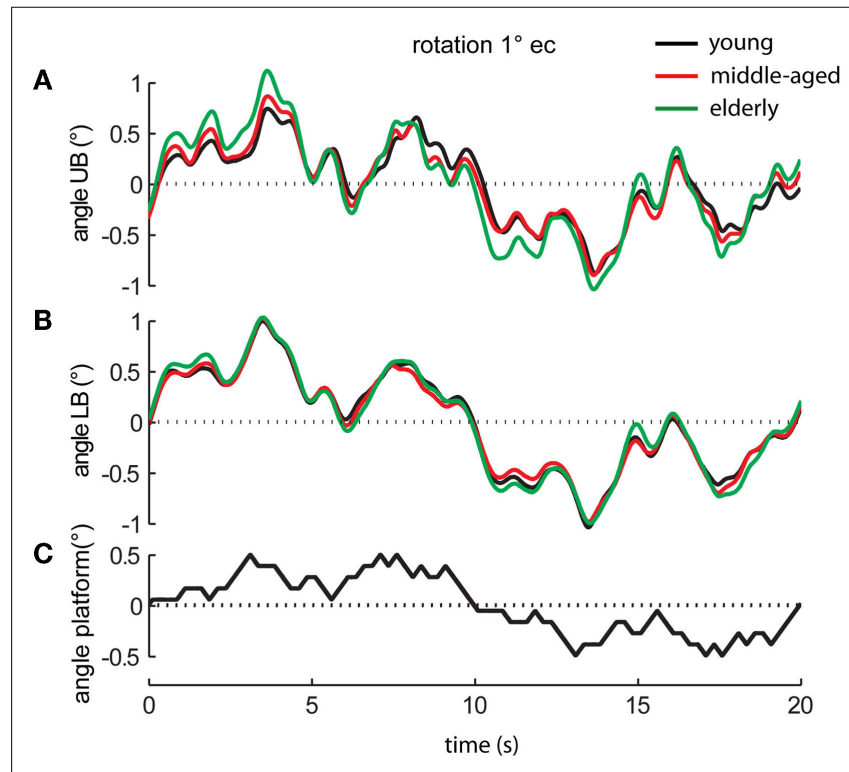

FIGURE 2 | Platform movements and motor reactions of the human body over time. Motor reactions of the three age groups' (A) upper body (UB) and (B) lower body (LB) at $1^{\circ}$ stimulus amplitude with eyes closed (ec) and (C) platform movement (black bottom line).

(Prieto et al., 1996; Maurer et al., 2003).

$$
\mathrm{RMS}=\mathrm{SD}=\sqrt{\frac{1}{n} \sum_{i=1}^{n}\left(x_{\mathrm{COP}}(i)\right)^{2}}
$$

Mean velocity is the average of the absolute COP velocity (Maurer and Peterka, 2005). It was calculated by differentiating the corresponding time series.

$$
\mathrm{MV}=\frac{1}{n-1} \sum_{\mathrm{i}=1}^{\mathrm{n}-1}\left|\dot{x}_{\mathrm{COP}}(i)\right|
$$

Mean frequency was computed as the ratio of MV and mean distance (MD).

$$
\text { MFREQ }=\frac{M V}{4 \cdot \sqrt{2} \cdot M D}
$$

Further details on spontaneous sway measures can be found in Maurer and Peterka (2005).

Transfer functions from stimulus-response data were calculated by a discrete Fourier transform (Peterka, 2002; Cnyrim et al., 2009). Fourier coefficients of stimulus and response time series are used to determine GAIN and PHASE with respect to stimulus frequencies (Maurer et al., 2006a). GAIN (response sensitivity) shows the relationship between the platform angle (stimulus amplitude) and the lower body or upper body response amplitude (Peterka, 2002; Maurer et al., 2006a; Cnyrim et al., 2009). PHASE is the relative delay between the stimulus and the reaction of the body. The transfer functions were used as the experimental data basis for model simulations using a predefined model of upright stance (see Figure 3).

Statistics were performed using Microsoft Excel and statistic programs (JMP ${ }^{\circledR}$ and Statview by SAS Institute Inc., Cary, NC,

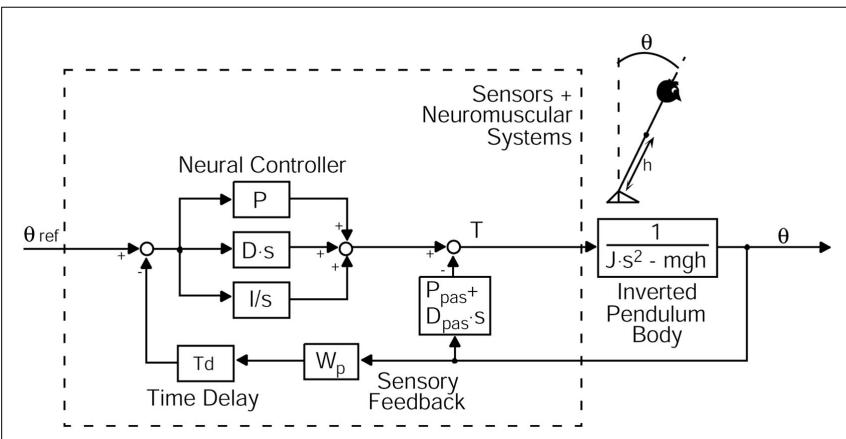

FIGURE 3 | Modified postural control model describing perturbed stance. The model consists of a body represented by an inverted pendulum with the mass concentrated at the center of mass (COM) of the body and the sensors and neuromuscular systems including a Neural Controller. $\theta$, body sway angle; h, height of the COM above the ankle joints; $\theta$ ref, external stimulus; P, proportional gain (stiffness factor), D, derivative gain (damping factor), I, integral gain of the neural controller; Ppas, passive stiffness factor; Dpas, passive damping factor; Wp, proprioceptive sensory weight; Td, feedback time delay; T, control torque; J, moment of inertia of the body; mgh, body mass $\times$ gravitational constant $\times$ height of the COM from the ankle joint; $\mathrm{s}$, Laplace transform variable.

USA). Statistical significance was tested by a two-level analysis of variance (ANOVA) unless stated otherwise. The between-subjects factor was age, the within-subjects factors were visual condition, sway direction, stimulus amplitude, stimulus frequency, and body segment (hip, shoulder). The level of statistical significance was set at $p=0.05$. For the elderly group, relationships between clinical test parameters and parameters obtained from platform experiments were analyzed using a Pearson Correlation [see Maurer and Peterka (2005)]. We created a matrix of correlation coefficients, which depicts the strength of linear relationships between each pair of parameters.

The study was performed according to the ethical standards of the Declaration of Helsinki. It was approved by the ethics committee of the University of Freiburg. All participants gave their written informed consent prior to study participation.

\section{Model Simulations}

We used a specific modification of an established postural control model (Van der Kooij et al., 2001; Mergner et al., 2002, 2003; Peterka, 2002; Maurer et al., 2004, 2006a; Cnyrim et al., 2009; Engelhart et al., 2014) to extract relevant parameters of postural control. This model includes a negative feedback loop that relates body excursion detected by visual, vestibular, and proprioceptive sensors to a corrective torque via a neural controller with proportional $[\mathrm{P}]$, derivative $[\mathrm{D}]$, and integral $[\mathrm{I}]$ contributions (PDI-controller, Figure 3). Neural controller gains are, in part, determined by mass and height of the COM of the individual subject [see Peterka (2002) and Cenciarini et al. (2010)]. Because our elderly group displayed lower masses and heights, we had to correct neural controller gains for this effect. That is why we give numbers for $[\mathrm{P} / \mathrm{mgh}],[\mathrm{D} / \mathrm{mgh}]$, and $[\mathrm{I} / \mathrm{mgh}]$, where $(\mathrm{mgh})$ represents the gravitational pull (body mass) $\times$ (gravitational constant $) \times$ (height of $\mathrm{COM}$ from the ankle joint $)$. [P/mgh] and [D/mgh] represent the stiffness and damping of our model. The proportional gain $[\mathrm{P} / \mathrm{mgh}]$, the derivative gain $[\mathrm{D} / \mathrm{mgh}]$, and the 
integral gain $[\mathrm{I} / \mathrm{mgh}]$ simulate the regulatory activity of the central nervous system to perturbations of stance (Nishihori et al., 2012).

Moreover, the model contains a lumped time delay [Td], which represents the time interval between the stimulus and the motor reaction. In addition, the model includes a sensory weighting mechanism [Wp], which represents the coordinate frame of the body excursion (visual and vestibular coordinates vs. platform coordinates). [Wp] describes the contribution of proprioception to the sensory feedback (Peterka, 2002). A decrease in [Wp] signifies that people tend to rely less on proprioception and more on vestibular and/or visual feedback. The biomechanics part represents the passive elasticity [Ppas] and passive damping factor [Dpas] of the muscles and tendons (Figure 3). With the help of an optimization procedure (fmincon/Matlab, Mathworks), we fit the model-derived transfer functions to the experimental transfer functions (GAIN and PHASE values) under different stimulus amplitudes and visual conditions. Subsequently, the set of model parameters representing the optimal fit were read out.

\section{Results}

\section{Spontaneous Sway}

The RMS of the elderly group $[0.56 \pm 0.011 \mathrm{~cm}$, (Mean \pm Standard Error)] was significantly larger than the RMS of the middleaged $(0.452 \pm 0.011 \mathrm{~cm})$ and young group $(0.447 \pm 0.011 \mathrm{~cm}$, $F=22.98, p<0.0001$, see Figure 4A for the COP-derived measures). Across all age groups, RMS was larger in the anterior-posterior $(\mathrm{a}-\mathrm{p})$ direction $(0.60 \pm 0.01 \mathrm{~cm})$ than in the mediolateral $(\mathrm{m}-\mathrm{l})$ direction $(0.38 \pm 0.01 \mathrm{~cm} ; F=187.5, p<0.0001)$, and significantly larger with eyes closed $(\mathrm{ec}, 0.52 \pm 0.01 \mathrm{~cm})$ than with eyes open (eo, $0.45 \pm 0.01 \mathrm{~cm} ; F=19.3, p<0.0001$ ). In general, RMS of the shoulder $(0.55 \pm 0.011 \mathrm{~cm})$ was significantly larger than RMS of the hip $(0.42 \pm 0.011 \mathrm{~cm}, F=66.12$, $p<0.0001)$. There were no significant interactions between age group and sway direction $(F=0.97, p=0.38)$, visual condition $(F=1.38, p=0.25)$, and body segments $(F=1.52, p=0.22)$.

As with RMS, MV of the elderly group was significantly higher $(0.7 \pm 0.018 \mathrm{~cm} / \mathrm{s})$ than MV of the middle-aged $(0.46 \pm 0.02 \mathrm{~cm} / \mathrm{s})$ and young group $(0.41 \pm 0.018 \mathrm{~cm} / \mathrm{s}, F=66.8, p<0.0001$, see Figure 4B for the COP-derived measures). Across all age groups, MV was larger in the $\mathrm{a}-\mathrm{p}$ direction $(0.65 \pm 0.015 \mathrm{~cm} / \mathrm{s})$ than in the $\mathrm{m}-\mathrm{l}$ direction $(0.4 \mathrm{~cm} / \mathrm{s} ; F=122.7, p<0.0001)$, and significantly larger with eyes closed $(0.6 \pm 0.015 \mathrm{~cm} / \mathrm{s})$ than with eyes open $(0.45 \pm 0.015 \mathrm{~cm} / \mathrm{s} ; F=38.7, p<0.0001)$. In addition, across all age groups, MV of the shoulder was significantly larger $(0.4 \pm 0.019 \mathrm{~cm} / \mathrm{s})$ than MV of the hip $(0.3 \pm 0.019 \mathrm{~cm} / \mathrm{s}, F=47.5$, $p<0.0001)$. Age group and sway direction significantly interacted, representing the fact that the MV difference between age groups was much larger in the $\mathrm{a}-\mathrm{p}$ than in the $\mathrm{m}-\mathrm{l}$ direction $(F=4.2$, $p=0.0153)$. Furthermore, there were no significant interactions between age group and visual condition $(F=1.93, p=0.146)$, or between age group and body segments $(F=1.3, p=0.285)$.

Effects on MF were similar to the effects on MV (see Figure 4C for the COP-derived measures). The MF of the elderly group was significantly higher $(0.3 \pm 0.007 \mathrm{~Hz})$ than the MF of the middleaged $(0.25 \pm 0.007 \mathrm{~Hz})$ and the young group $(0.24 \pm 0.007 \mathrm{~Hz}$, $F=16.97, p<0.0001)$. Across all age groups, MF was larger in
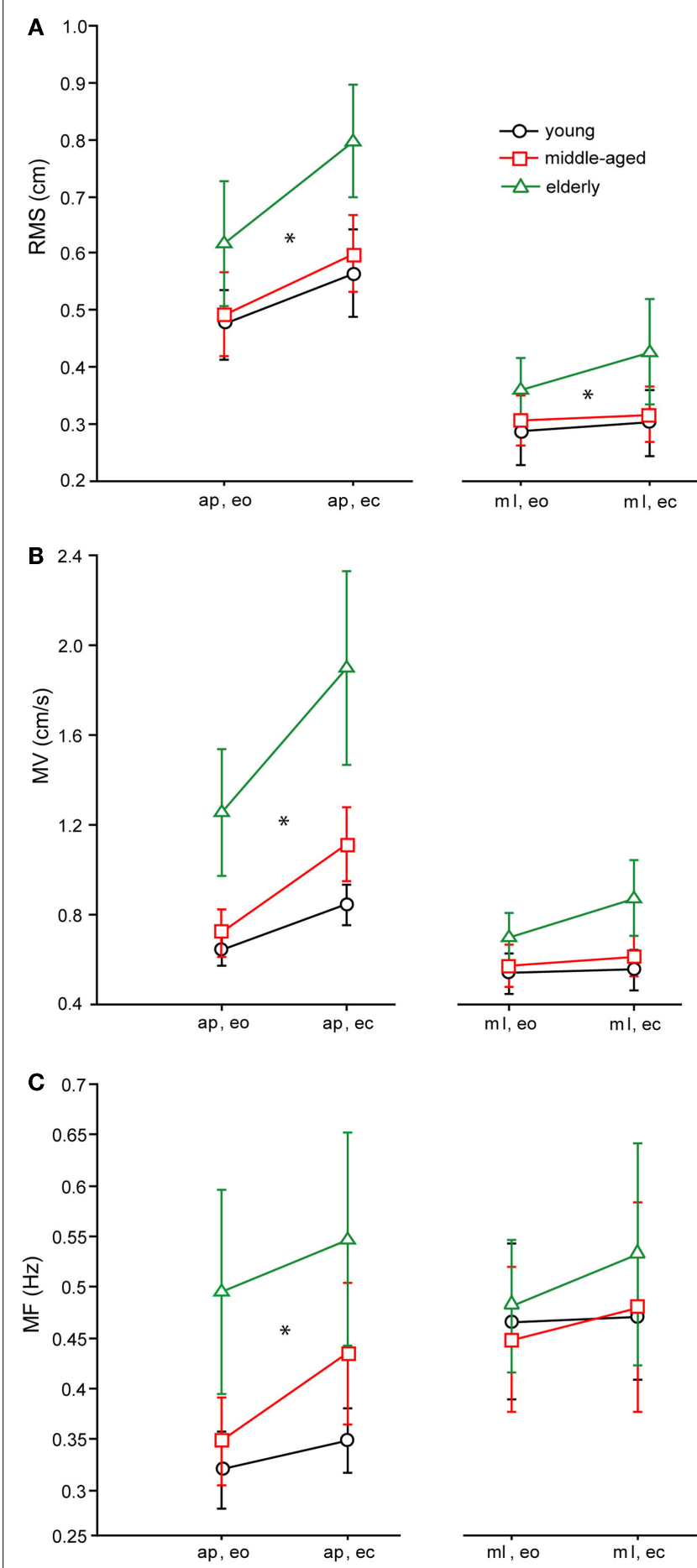

FIGURE 4 | Spontaneous sway parameters derived from COP traces. (A) Root mean square (RMS) of the three age groups, (B) mean velocity (MV) of the THREE age groups, (C) mean frequency (MF) of the three age groups. a-p, anterior-posterior; ml, medio-lateral; eo, eyes open; ec, eyes closed.

the $\mathrm{m}-\mathrm{l}$ direction $(0.28 \pm 0.006 \mathrm{~Hz})$ than in the $\mathrm{a}-\mathrm{p}$ direction $(0.25 \pm 0.006 \mathrm{~Hz}, F=3.4, p=0.035)$, and significantly larger with eyes closed $(0.28 \pm 0.006 \mathrm{~Hz})$ than with eyes open $(0.25 \mathrm{~Hz}$; $F=9.18, p=0.003)$. In general, MF of hip $(0.17 \pm 0.007 \mathrm{~Hz})$ 
and shoulder $(0.17 \pm 0.007 \mathrm{~Hz})$ were nearly equal, which was not statistically significant $(F=0.2, p=0.620)$. Age group and sway direction significantly interacted $(F=3.4, p=0.035)$, representing the fact that the MF difference between age groups was much larger in the $\mathrm{a}-\mathrm{p}$ than in the $\mathrm{m}-\mathrm{l}$ direction. In addition, we found no significant interaction between age group and body segments $(F=0.1, p=0.887)$ or between age group and visual condition $(F=1.9, p=0.157)$.

\section{Externally Perturbed Stance}

We characterize the participants' sway behavior as a function of external perturbation by a transfer function in the frequency domain over 11 frequencies $(0.05-2.2 \mathrm{~Hz}$, see Procedures). The transfer function consists of a gain and a phase curve. For didactic reasons, we display GAIN effects of the middle-aged and elderly group also as GAINFACTOR with respect to a reference group (young group). GAINFACTOR is the percentage gain of the two elder groups with respect to the young reference group.

We found significant differences in GAIN between the three age groups $(F=327.5, p<0.0001)$. In the young group, GAIN was lowest $(1.66 \pm 0.022)$ whereas it was highest in the elderly group $(2.42 \pm 0.021)$. Across all groups, GAIN was on average $37.6 \%$ higher with eyes closed than with eyes open $(F=621.2$, $p<0.0001)$. GAIN significantly depended on stimulus amplitudes $\left(0.5^{\circ}: 2.21 \pm 0.018,1^{\circ}: 1.80 \pm 0.018, F=249.3, p<0.0001\right)$, on stimulus frequencies $(F=394.9, p<0.0001)$, and on body segments (hip: $1.62 \pm 0.018$, shoulder: $2.40 \pm 0.018, F=878.3$, $p<0.0001)$. Age group significantly interacted with frequency $(F=9.52, p<0.0001)$. The major GAIN difference between age groups appeared to be in the lower frequency range, except for the lowest frequency value (see GAIN and GAINFACTOR plots in Figures 5 and 6). Moreover, age group significantly interacted with body segments $(F=170.7, p<0.0001)$. This represents the fact that GAIN of the shoulder in the elderly group was almost twice as large as that of the hip, whereas in the young group, shoulder GAIN was 20\% larger than hip GAIN (Figure 7A). Finally, age group significantly interacted with visual conditions $(F=3.17, p=0.042)$, representing the finding that GAIN with eyes closed was about $50 \%$ larger than with eyes open in the young group, whereas the GAIN increase with closing the eyes was only $25 \%$ in the elderly group (Figure $7 \mathbf{B}$ ). There was no significant interaction between age group and stimulus amplitude $(F=0.9$, $p=0.39$ ).

Across all age groups, PHASE was mainly determined by frequency $(F=849.2, p<0.0001)$. PHASE differed significantly between the three age groups (young: $-123.45 \pm 1.52^{\circ}$, middleaged: $\quad-131.82 \pm 1.65^{\circ}$, elderly: $\quad-122.77 \pm 1.48^{\circ} ; \quad F=9.9$, $p=<0.0001)$. More specifically, the PHASE profile as a function of frequency was different between the age groups. While the young group showed a moderate slope of the PHASE as a function of stimulus frequencies, the middle-aged and the elderly group presented a steeper relationship between PHASE and frequencies (see Figures 5 and 6). Interestingly, the PHASE profile difference between the middle-aged and the elderly group mainly consisted of a downward shift (reduction of phase lag) that was consistent across all frequencies (LAGPHASE plots in Figures 5 and 6). In general, the effect of age on PHASE as a function of frequency was characterized by a significant interaction between age and frequency $(F=3.5, p<0.0001)$. Across all age groups, phase lag was found to be significantly smaller with eyes closed $\left(-121.82 \pm 1.27^{\circ}\right)$ than with eyes open $\left(-129.44 \pm 1.27^{\circ}, \quad F=17.6, \quad p<0.0001\right)$, significantly smaller at the hip $\left(-101.26 \pm 1.27^{\circ}\right)$ than at the shoulder level $\left(-150.01 \pm 1.27^{\circ}, F=761.5, p<0.0001\right)$, but was not significantly different across different stimulus amplitudes $(F=0.02, p=0.87)$. Age group significantly interacted with body segment $(F=27.1$, $p<0.0001)$ representing the fact that phase difference between shoulder and hip decreases with age (see Figure 7C). There was no significant interaction between age group and visual condition $(F=0.5, p=0.6002)$.

\section{Model Parameters}

The model parameter/stiffness factor $[\mathrm{P} / \mathrm{mgh}]$ is the proportional gain of the neural controller. We found significant differences of $[\mathrm{P} / \mathrm{mgh}]$ across the three age groups $(F=9.3, p=0.0001$, see Figure 8A). This parameter was highest in the young group $\left(1.43 \mathrm{rad}^{-1}\right)$ and lowest in the elderly group (1.29 $\left.\mathrm{rad}^{-1}\right)$. Across all age groups, $[\mathrm{P} / \mathrm{mgh}]$ was significantly higher at a stimulus amplitude of $1^{\circ}\left(1.41 \mathrm{rad}^{-1}\right)$ than of $0.5^{\circ}\left(1.33 \mathrm{rad}^{-1}, F=6.7\right.$, $p=0.01$ ), representing a slight amplitude non-linearity of the system. Visual conditions did not significantly influence $[\mathrm{P} / \mathrm{mgh}]$ $(F=0.7, p=0.42)$. The age group did not significantly interact with visual condition $(F=0.9, p=0.39)$ or stimulus amplitude $(F=0.02, p=0.98)$.

The derivative gain or damping factor, [D/mgh], did not significantly vary with age $(F=0.1, p=0.87$, Figure 8A). This parameter was nearly equal in the three age groups (young: $0.372 \mathrm{~s} \times \mathrm{rad}^{-1}$, middle-aged: $0.370 \mathrm{~s} \times \mathrm{rad}^{-1}$, elderly: $\left.0.365 \mathrm{~s} \times \mathrm{rad}^{-1}\right)$. ([D/mgh] was significantly higher with eyes closed $\left(0.37 \mathrm{~s} \times \mathrm{rad}^{-1}\right)$ than with eyes open $\left(0.35 \mathrm{~s} \times \mathrm{rad}^{-1}, F=9.34, p=0.0025\right)$. Stimulus amplitude had a significant effect on $[\mathrm{D} / \mathrm{mgh}]\left(0.5^{\circ}\right.$ : $\left.0.36 \mathrm{~s} \times \mathrm{rad}^{-1}, 1^{\circ}: 0.38 \mathrm{~s} \times \mathrm{rad}^{-1}, F=4.4, p=0.04\right)$. The age group did not have a significant interaction with visual condition $(F=0.47, p=0.62)$ or stimulus amplitude $(F=0.23, p=0.79)$ considering their effect on $[\mathrm{D} / \mathrm{mgh}])$.

The integral gain, $[\mathrm{I} / \mathrm{mgh}]$, was significantly higher in the young and the middle-aged group (young: $0.118 \mathrm{~s}^{-1} \times \mathrm{rad}^{-1}$, middle-aged: $\left.0.120 \mathrm{~s}^{-1} \times \mathrm{rad}^{-1}\right)$ than in the elderly group $\left(0.097 \mathrm{~s}^{-1} \times \mathrm{rad}^{-1}, F=9.4, p=0.0001\right.$, Figure 8A). Visual condition had a significant effect on $[\mathrm{I} / \mathrm{mgh}]\left(\right.$ eo: $0.118 \mathrm{~s}^{-1} \times \mathrm{rad}^{-1}$, ec: $\left.0.104 \mathrm{~s}^{-1} \times \mathrm{rad}^{-1}, F=8.8, p=0.0033\right)$, while stimulus amplitude did not $(F=0.02, p=0.88)$. The age was found to interact significantly with visual condition $(F=1.1, p=0.34)$ or stimulus amplitude $(F=0.2, p=0.86)$ in their effect on [I/mgh].

The age group was found to have a significant effect on the passive stiffness factor [Ppas/mgh] $(F=9.9, p<0.0001$, see Figure 8B). [Ppas/mgh] was smallest in the elderly group (0.109) and larger in the other two age groups (young: 0.130, middle-aged: 0.135). [Ppas/mgh] was significantly higher with eyes open $(0.135)$ than with eyes closed $(0.113, F=19.02, p<0.0001)$. Stimulus amplitude did not significantly influence [Ppas/mgh] $(F=0.6$, $p=0.45)$. The age group was not found to significantly interact with visual condition $(F=0.5, p=0.62)$ or stimulus amplitude $(F=0.3, p=0.72)$ with respect to [Ppas/mgh].

[Dpas/mgh], the passive damping factor, decreased significantly with age $(F=9.07, p=0.0002$, Figure 8B). It was highest in 

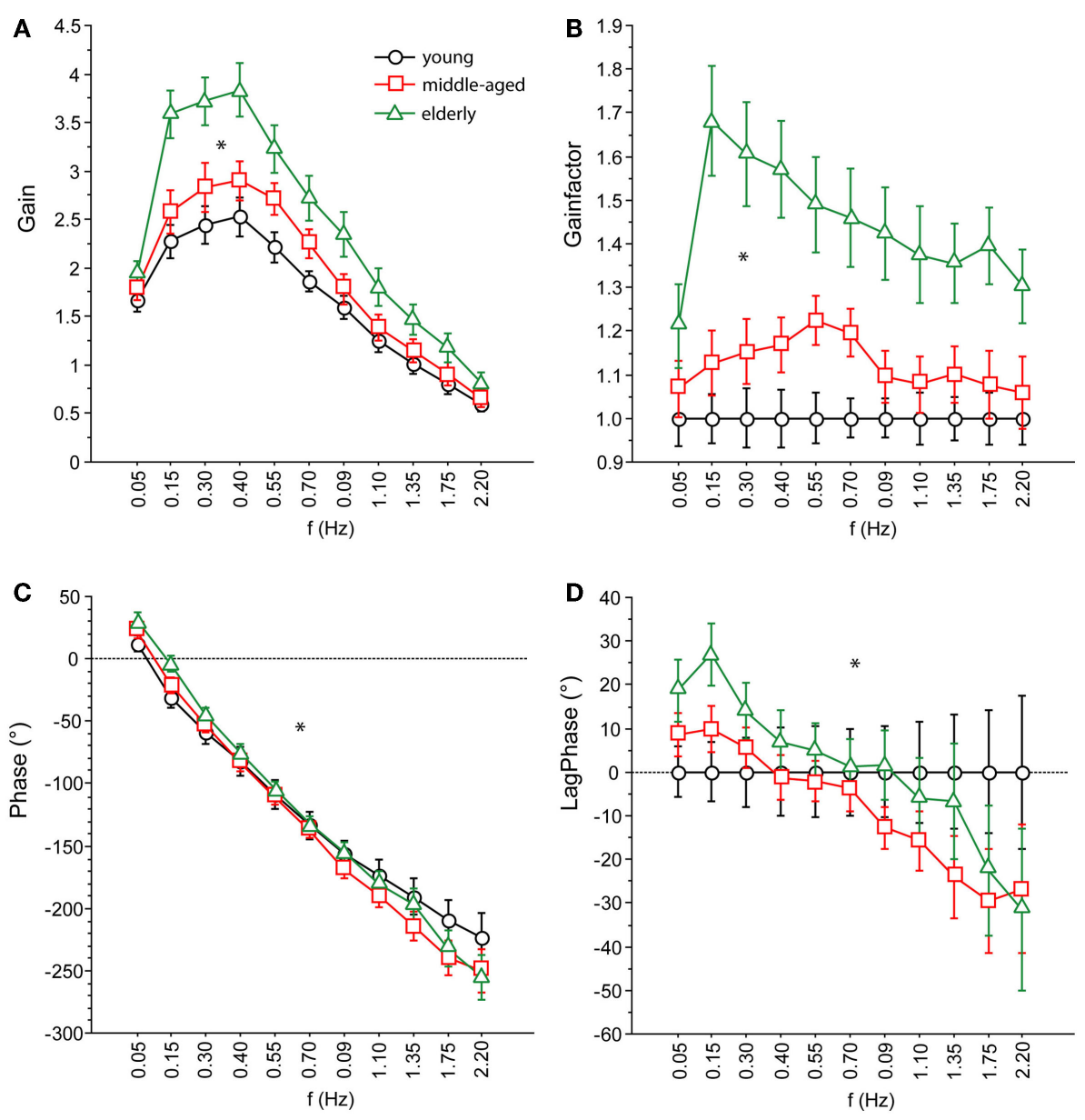

FIGURE 5 | Parameters of perturbed stance. (A) GAIN, (B)

GAINFACTOR, (C) PHASE, (D) LAGPHASE of the three age groups across all stimulus amplitudes and visual conditions. GAINFACTOR is the percental quotient between the GAIN of the middle-aged and elderly group and the young group as a comparison group. The time difference between PHASE of the middle-aged and elderly group and the young group as a comparison group is named LAGPHASE. Its unit is degrees $\left({ }^{\circ}\right)$. the young group (0.089) and lowest in the elderly group (0.074). [Dpas/mgh] was significantly higher with eyes open (0.089) than with eyes closed $(0.078, F=10.9, p=0.0011)$. Stimulus amplitude had no significant influence on [Dpas/mgh] $(F=0.3, p=0.61)$. The age group was not observed to have a significant interaction with visual condition $(F=0.14, p=0.87)$ or stimulus amplitude $(F=0.1, p=0.89)$ with respect to [Dpas/mgh].

Time delay, [Td], significantly increased with age ( $F=14.1, p<0.0001$, Figure 8C). It was lowest in the young group $(0.153 \pm 0.003 \mathrm{~s})$ and highest in the elderly group $(0.177 \pm 0.003 \mathrm{~s})$. Neither visual condition $(F=0.9, p=0.34)$ nor stimulus amplitude $(F=2.9, p=0.09)$ had a significant effect on [Td]. Moreover, age group did not significantly interact with visual condition $(F=0.2, p=0.85)$ or stimulus amplitude $(F=0.88, p=0.42)$ with respect to [Td].

The proprioceptive sensory weight, $[\mathrm{Wp}]$, increased significantly with increasing age $(F=7.2, p=0.0009$, see Figure 8D). Being lowest in the young group $(0.64 \pm 0.018)$ and highest in the elderly group $(0.74 \pm 0.018)$, [Wp] was $35 \%$ higher with eyes closed $(0.8 \pm 0.015)$ than with eyes open $(0.59 \pm 0.015)$, and this difference was significant $(F=93.5, p<0.0001)$. The stimulus amplitude significantly affected [Wp] $\left(0.5^{\circ}: 0.74 \pm 0.015\right.$, $\left.1^{\circ}: 0.65 \pm 0.015, F=18.7, p<0.0001\right)$, resulting in a $14 \%$ increase. The age group did not significantly interact with visual condition $(F=1.8, p=0.16)$ or stimulus amplitude $(F=0.3, p=0.77)$ in their effect on $[\mathrm{Wp}]$. In addition to [Wp], we show a figure of [Wspace] (Figure 8E). [Wspace] is 1-[Wp] and it reflects vestibular weight with eyes closed and visual and vestibular weight with eyes open.

\section{Clinical Tests}

The elderly group performed also two clinical tests: the TUG and the FRT. The average results were for the FRT a reach distance of $28.2 \pm 6.4 \mathrm{~cm}$ and for the TUG $8.48 \pm 1.2 \mathrm{~s}$.

In the following, we report significant correlations between our experimental spontaneous sway measures and measures of perturbed stance, derived model parameters, and the results of clinical tests in the elderly group (see Figure 9; Table 1). However, we calculated the correlation matrix only between measures (spontaneous and perturbed sway measures) and parameters that 

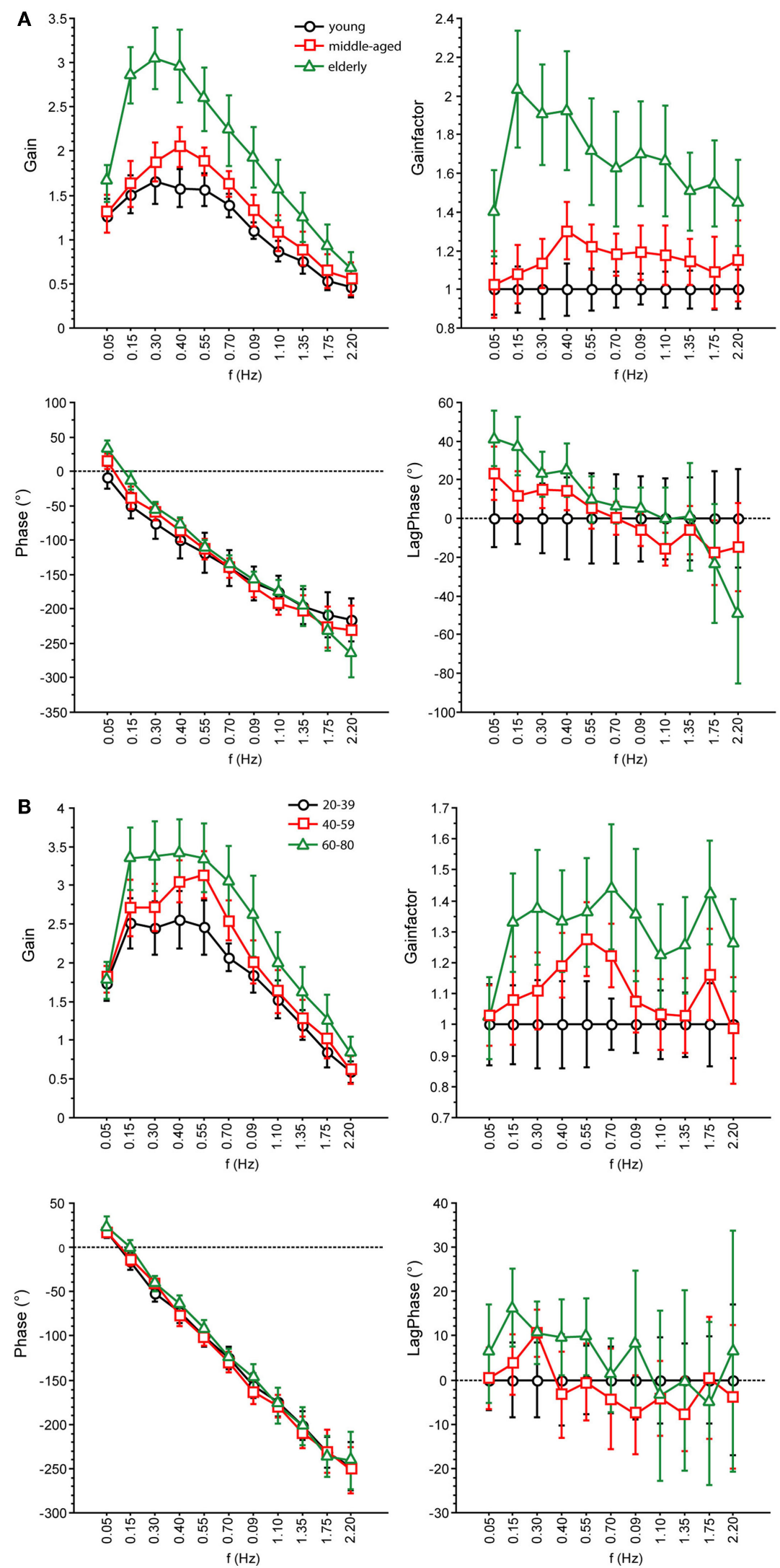

FIGURE 6 | Parameters of perturbed stance. GAIN, GAINFACTOR, PHASE, LAGPHASE of the three age groups at $1^{\circ}$ with eyes open (A) and eyes closed (B). 

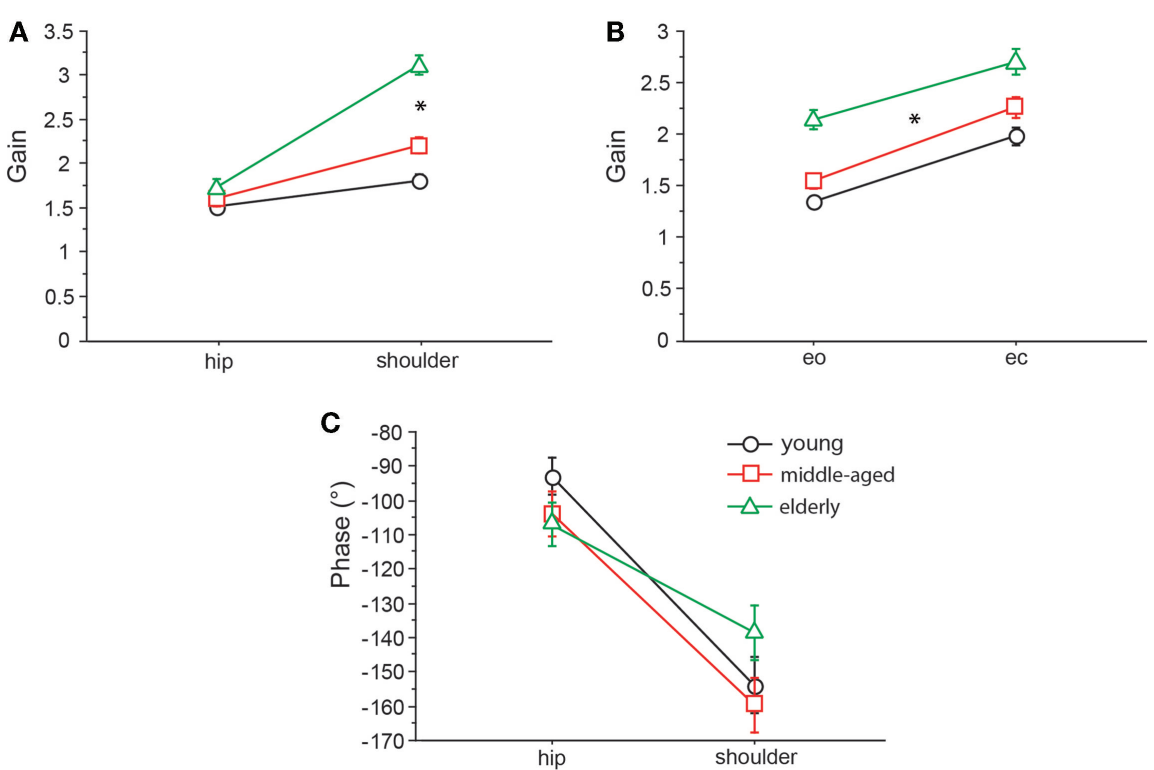

FIGURE 7 | GAIN interaction between age and body segments and age and visual condition, and PHASE interaction between age and body segments. GAIN of the three age groups with respect to body segment (A) and visual condition (B) and PHASE of the three age groups with respect to body segment (C). eo, eyes open; ec, eyes closed. were significantly different from young subjects. PHASE, MF, $\mathrm{MV}$, [P/mgh], and TUG all correlated significantly with each other. In addition, PHASE correlated significantly with [Td], [Td] correlated with [Wp], [Wp] correlated with GAIN and GAIN correlated with TUG. By contrast, RMS correlated only with MF and FRT correlated only with TUG.

\section{Discussion}

We analyzed postural control of elderly people (60-80 years) and compared their data to the data of two younger groups (20-39 and 40-59 years). Postural control was characterized by spontaneous sway measures and measures of perturbed stance between 0.05 and $2.2 \mathrm{~Hz}$. Perturbations were induced by a pseudorandom platform tilt stimulus. Stimulus-response data were interpreted on the basis of a simple negative feedback model (Peterka, 2002; Maurer et al., 2006a; Engelhart et al., 2014).

Among the spontaneous sway measures, RMS, MV, and MF were significantly higher in elderly people (elderly group) than in young people (young group). These results are in line with previous studies (Maki et al., 1990; Hytönen et al., 1993; Baloh et al., 1994; Collins et al., 1995; Prieto et al., 1996; Tang and Woollacott, 1996; Maurer and Peterka, 2005). The effect on MV had the highest significance. The high sensitivity of the MV effect as compared to other spontaneous sway measures to detect abnormalities was reported earlier (Prieto et al., 1996; Maurer and Peterka, 2005; Ruhe et al., 2010; Delignières et al., 2011; Moghadam et al., 2011). In previous work by our group (Maurer and Peterka, 2005) and others (Maki et al., 1990; Prieto et al., 1996), higher MV of elderly people was interpreted as a higher amount of regulatory balancing activity. Furthermore, MV was different from other spontaneous sway parameters as the age effect significantly interacted with sway direction ( $a-p$ vs. $m-1)$. The interaction with sway direction was based on the fact that elderly's $\mathrm{MV}$ in the a-p direction was much larger $(+36 \%)$ than in $\mathrm{m}-1$ direction, whereas this directional effect was much weaker in the younger groups. As already shown in other studies, a-p sway might be more sensitive to any impairment (Collins et al., 1995; Prieto et al., 1996; Tia et al., 2012), due to the fact that balance in $a-p$ direction follows the rules of an unstable inverted pendulum.

Mean velocity of the middle-aged and elderly group increased about 30\% (elderly: 26\%, middle-aged 36\%) across all body segments when closing the eyes. In the young group, this increase amounted to only $18 \%$. Independently of age, the increase of MV when closing the eyes has been described before [e.g., Era and Heikkinen (1985), Horak et al. (1989), Teasdale et al. (1991), Hytönen et al. (1993), Prieto et al. (1996), Accornero et al. (1997), Schieppati et al. (1999), and Shumway-Cook and Woollacott (2001)]. It is still controversial whether this increase is more or less pronounced in elderly than in young subjects. Peterka and Black (1990) and Teasdale et al. (1991) observed as well no significant age-related increase in spontaneous sway with eyes closed. Hytönen et al. (1993) found an increased difference of sway velocity between eyes closed and eyes open in their eldest group (76-90 years).

In general, spontaneous sway measures in our study seemed to mirror age-dependent changes of postural control in a reliable way. However, the variance across scores of different spontaneous sway measures is known to be covered by only two principal components, even if one analyzes up to 14 different measures [see Maurer and Peterka (2005)]. That might be the reason why measuring spontaneous sway does not allow for detecting specific constituents of postural control deficits (Horak et al., 1989; Maki et al., 1990; Tang and Woollacott, 1996; 

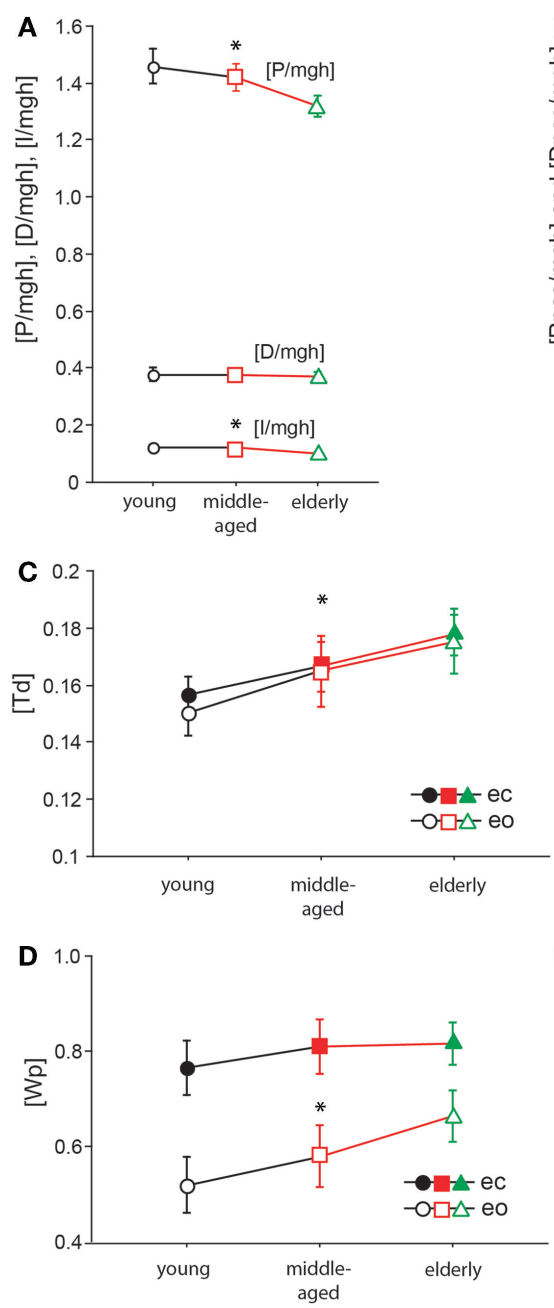
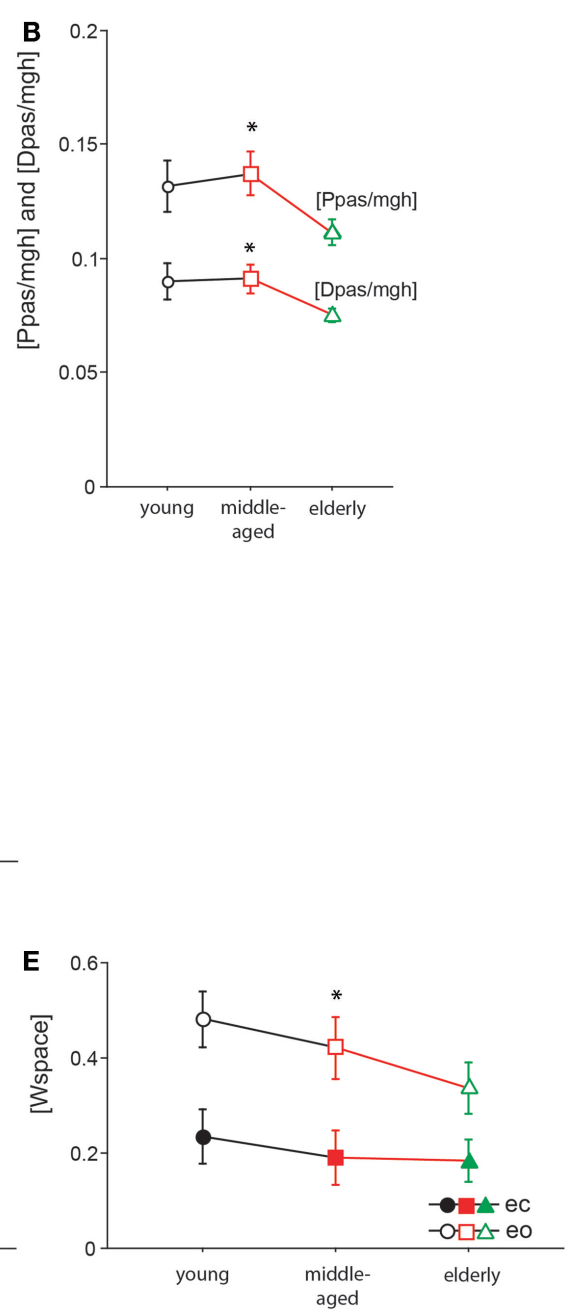

FIGURE 8 | Model parameters. [P/mgh] in $\mathrm{rad}^{-1}$ (A), [D/mgh] in $\mathrm{s} \times \mathrm{rad}^{-1}(\mathbf{A}),[\mathrm{l} / \mathrm{mgh}] \mathrm{in} \mathrm{s}^{-1} \times \mathrm{rad}^{-1}(\mathbf{A}),[\mathrm{Ppas} / \mathrm{mgh}] \mathbf{( B )}$, [Dpas/mgh] (B), [Td] in s (C), [Wp] (D), and [Wspace] (E) of the three age groups. The three different age groups are shown on the $x$-axis; eo, eyes open; ec, eyes closed.

Kuo et al., 1998; Black, 2001; Ghulyan et al., 2005). From a model-based perspective, many different sources of postural control deficits like, e.g., increased feedback time delay, too strong or too weak feedback gain, increased sensory noise level, and abnormal weighting of sensory inputs may all lead to increased sway or sway velocity (Maurer and Peterka, 2005). For a more specific analysis of postural control deficits, an external perturbation is required (Engelhart et al., 2014). Consequently, we characterized subjects' behavior as a function of external perturbations, i.e., anterior-posterior platform tilts, by transfer functions in the frequency domain. Transfer functions consist of gain and phase curves. We first discuss GAIN and PHASE findings separately before we integrate the findings using our model-based approach.

GAIN was highest in elderly and lowest in young people, which is in line with earlier findings [e.g., Ghulyan et al. (2005) during sinusoidal platform translations]. Across all age groups, GAIN significantly depended on visual conditions, stimulus amplitudes, stimulus frequencies, and on body segments. Moreover, we found significant interactions between age, on one hand, and frequency, visual condition, and body segments, on the other. The interaction between age and frequency represents the fact that the major GAIN difference between age groups appeared in the lower frequency range. The interaction between age and visual condition is due to the fact that the GAIN increase was about $48 \%$ when closing the eyes in the youngest group and only $26 \%$ in the elderly group. The significant interaction between age and body segments is related to the relatively larger shoulder GAIN (almost twice as large as the hip GAIN) in elderly people, whereas in young people, shoulder GAIN was $20 \%$ larger than hip GAIN. This finding is in line with the fact that elderly people are known to have a tendency to engage hip flexion/extension when stance is perturbed (Kuo et al., 1998; Shumway-Cook and Woollacott, 2001).

PHASE as a function of frequency was significantly different between the three age groups. The PHASE decreased with increasing frequency in all age groups; however, the middle-aged and the elderly group displayed a steeper relationship between PHASE and frequencies. Age significantly interacted with body segment, representing the fact that PHASE differences between shoulder and hip decreases with age. 
In order to interpret the numerous findings concerning GAIN and PHASE curves, we fitted subjects' data by a simple feedback system that is known to adequately describe body motion as a function of sensory inputs [e.g., Mergner et al. (2002, 2003), Vette et al. (2010), Van der Kooij and Peterka (2011), and Engelhart et al. (2014)]. A very basic version consists of the body represented by mass and height, a neural controller including stiffness factor and damping factor, a feedback time delay, and sensory weighting mechanism that integrates proprioceptive, vestibular, and visual cues (Maurer et al., 2006a; Van der Kooij and Peterka, 2011). We had to reject our hypothesis that elderly people show a general

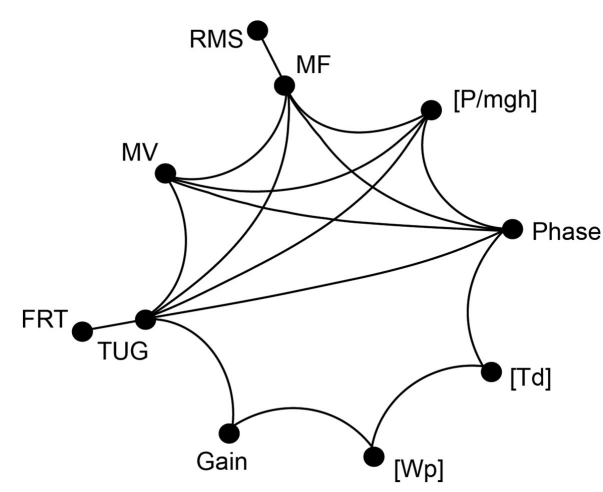

FIGURE 9 | Correlation of clinical tests and measurements of spontaneous and perturbed stance. Correlation matrix between clinical test parameters and parameters obtained from platform measures. The correlation revealed a group of parameters that all significantly correlate with each other (MF, MV, PHASE, [P/mgh], and TUG). Some of the measures mentioned above significantly correlate with additional measures and parameters. Only correlations with $R>0.47$ and $p<0.05$ are shown. decline in sensory, motor, and higher-level adaptation systems. The most significant differences between age groups relate to the sensory weighting, the feedback gain of the neural controller, and the overall time delay of the motor reaction: Across all stimulus conditions, elderly people weigh proprioceptive cues ([Wp]) higher than visual and vestibular cues. This finding suggests that elderly people tend to stabilize and orient their body relative to the support surface and, therefore, rely more on proprioceptive than on vestibular or visual cues, which is related to the larger GAIN across all frequencies. Abnormalities in the use of sensory cues have already been reported by others (Nishihori et al., 2012; Maitre et al., 2013). All subjects weigh proprioceptive cues relatively stronger when they close their eyes, i.e., one space reference cue (visual) is missing. This relates to the larger GAIN observed with eyes closed than with eyes open. The vestibular cue as another space cue does not fully compensate for the lack of the visual cue as also reported by others (Ishida et al., 1997). This is in line with the experience of other laboratories that other cues fill in when sensory cues are missing (Van der Kooij and Peterka, 2011).

Although not being significant, elderly people tend to downregulate proprioceptive cues when opening the eyes less than young people do. Then again, all age groups similarly downregulate their proprioceptive weights with increasing stimulus amplitude, representing the fact that with larger disturbances of the support surface, stabilization in space becomes advantageous to avoid large body sway (Peterka, 2002; Van der Kooij and Peterka, 2011). This might indicate that the reliability of the sensory weighting process is not impaired in elderly people as a result of aging.

Elderly people display an overall time delay that is about $24 \mathrm{~ms}$ longer than that of young people. Our results are almost identical to the results of Davidson et al. (2011) in which the time delay of

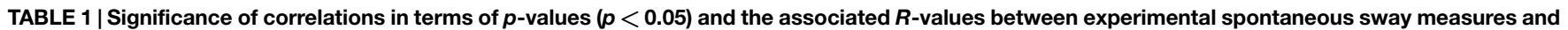
measures of perturbedstance, derived model parameters, and the results of the clinical tests in the elderly group.

\begin{tabular}{|c|c|c|c|c|c|c|c|c|c|c|}
\hline & Phase & [P/mgh] & MF & RMS & MV & TUG & FRT & GAIN & [Wp] & [Td] \\
\hline $\begin{array}{l}\text { Phase } \\
\text { [P/mgh] }\end{array}$ & $\begin{array}{c}p=0.0008 \\
R=0.72\end{array}$ & & & & & & & & & \\
\hline MF & $\begin{array}{c}p=0.0001 \\
R=0.79\end{array}$ & $\begin{array}{c}p=0.0001 \\
R=0.88\end{array}$ & & & & & & & & \\
\hline RMS & n.s. & n.s. & $\begin{array}{l}p=0.0307 \\
R=-0.51\end{array}$ & & & & & & & \\
\hline MV & $\begin{array}{c}p=0.0012 \\
R=0.70\end{array}$ & $\begin{array}{c}p=0.0028 \\
R=0.66\end{array}$ & $\begin{array}{c}p=0.0052 \\
R=0.63\end{array}$ & n.s. & & & & & & \\
\hline TUG & $\begin{array}{c}p=0.0369 \\
R=0.49\end{array}$ & $\begin{array}{c}p=0.002 \\
R=0.68\end{array}$ & $\begin{array}{c}p=0.0353 \\
R=0.50\end{array}$ & n.s. & $\begin{array}{c}p=0.0464 \\
R=0.47\end{array}$ & & & & & \\
\hline FRT & n.s. & n.s. & n.s. & n.s. & n.s. & $\begin{array}{l}p=0.0433 \\
R=-0.48\end{array}$ & & & & \\
\hline GAIN & n.s. & n.s. & n.s. & n.s. & n.s. & $\begin{array}{c}p=0.0461 \\
R=0.48\end{array}$ & n.s. & & & \\
\hline [Wp] & n.s. & n.s. & n.s. & n.s. & n.s. & n.s. & n.s. & $\begin{array}{c}p=0.0001 \\
R=0.78\end{array}$ & & \\
\hline$[\mathrm{Td}]$ & $\begin{array}{c}p=0.0343 \\
R=-0.51\end{array}$ & n.s. & n.s. & n.s. & n.s. & n.s. & n.s. & n.s. & $\begin{array}{c}p=0.0331 \\
R=0.50\end{array}$ & \\
\hline
\end{tabular}

n.s., not significant; MF, mean frequency; RMS, root mean square; TUG, timed up and go test; FRT, functional reach test; Td, time delay. 
the motor reaction in elderly people was found to be $23 \mathrm{~ms}$ higher than in the young people when perturbing stance with jolts of a ballistic pendulum. A large time delay endangers stability of the system through an enlarged oscillation tendency (Peterka, 2000; Van der Kooij and Peterka, 2011). Since a high-proportional feedback gain increases oscillation tendencies, too, a lower feedback gain in elderly might represent a compensation strategy to reduce oscillations (Peterka, 2002; Van der Kooij and Peterka, 2011). In fact, we identified smaller feedback gain parameters (active proportional, [P/mgh]; passive proportional, [Ppas/mgh]; passive damping factor, [Dpas/mgh]) in elderly compared to young subjects.

Everyday postural control is usually assessed by simple clinical tests and scales such as the TUG (Podsiadlo and Richardson, 1991; Enkelaar et al., 2013) and the FRT (Duncan et al., 1990; Enkelaar et al., 2013). In order to relate our findings to established measures of postural control, our elderly subjects performed FRT and TUG. The FRT revealed an average reach distance of $28.2 \mathrm{~cm}$ in the elderly group. The reach distance of our elderly group is similar to data of Duncan et al. (1990) who evaluated the FRT in 128 volunteers between 21 and 87 years. Their group of elderly people aged between 70 and 87 years attained a reach distance of $25.0 \mathrm{~cm}$ whereas the younger group (20-40ys) showed a distance of $39.8 \pm 5.2 \mathrm{~cm}$. In our study, the TUG in the elderly group amounted to $8.48 \mathrm{~s}$. The TUG scores of the elderly group are similar to the one reported by Buatois et al. (2006); (9.6-10.2 s), Nagy et al. (2007); (8.9-10.3 s), Enkelaar et al. (2013); (9.3 s), and Bohannon (2006); (9.2 s).

The correlation analysis between clinical scales (FRT, TUG) and our experimental findings in the elderly group revealed a group of parameters that all significantly correlated with each other, consisting of two spontaneous sway measures (MF, MV), one measure of perturbed stance (PHASE), one model-derived parameter $([\mathrm{P} / \mathrm{mgh}])$, and the clinical measure of TUG (see Table 1). This could be explained by the fact that all those parameters may be influenced by systems stability. Why would a clinical score like TUG, which involves walking and transitioning between straight walking, turning, sit-to-stand and stand-to-sit motions, correlate with parameters that describe human postural control? We assume that stability of the postural control system also affects more complex postural tasks such as walking or certain transitions.

Furthermore, there was a significant correlation between PHASE and [Td], which are both related to the timing of a response following a stimulus. [Wp] correlated significantly with

\section{References}

Accornero, N., Capozza, M., Rinalduzzi, S., and Manfredi, G. W. (1997). Clinical multisegmental posturography: age-related changes in stance control. Electroencephalogr. Clin. Neurophysiol. 105, 213-219. doi:10.1016/S0924-980X(97) 96567-X

Allison, L. K., Kiemel, T., and Jeka, J. J. (2006). Multisensory reweighting of vision and touch is intact in healthy and fall-prone older adults. Exp. Brain Res. 175, 342-352. doi:10.1007/s00221-006-0559-7

Baloh, R. W., Fife, T. D., Zwerling, L., Socotch, T., Jacobson, K., Bell, T., et al. (1994). Comparison of static and dynamic posturography in young and older
GAIN and this is consistent with [Wp] being responsible for a uniform scaling of the gain curves across all frequencies.

It was interesting to find that the FRT correlated only with the TUG. The fact that the FRT seems to correlate with other clinical balance measures has been reported by others (Granacher et al., 2009; Enkelaar et al., 2013: moderate correlation). However, it is not clear why a stance parameter related to the amount of forward voluntary lean is related to a complex dynamic movement task as the TUG. We deem it likely, however, that the endangered stability of the postural control system described above, might influence both, voluntary lean and more complex movement tasks. Further investigations are needed to explain this finding. Furthermore, RMS correlated significantly solely with MF, while MF correlated with many other measures of perturbed sway analogously to MV, supporting our view that spontaneous sway measures are gross measures related to balance control and that the information contained in those measures is highly correlated and possibly redundant (Maurer and Peterka, 2005). More specifically, sway amplitude, represented by RMS, might be less crucial for the postural stability than velocity related measures such as MV and MF.

In summary, the perturbation-based approach presented here gives us more insight about postural control mechanisms of elderly people than measures of spontaneous sway do. In addition, it allows for a model-based interpretation of the experimental data, which provide valuable information of underlying mechanisms of the whole postural control system. We were able to identify basic parameters of the postural control system that are related to aging, i.e., the increased reliance of elderly people on proprioception, the decreased feedback loop gain, and the increased closed loop feedback time delay between the sensory perception and the motor reaction. Understanding the postural changes during aging is crucial for the development of new therapies that improve postural control in the elderly population. Our approach is highly sensitive even to small changes in postural control. Accordingly, it might be also suited to monitor therapeutic interventions, which aim to improve postural stability in the elderly.

\section{Acknowledgments}

We would like to thank Anja Wehrle and Jörg Dietterle for their support during the measurements. In addition, we thank Dr. Massimo Cenciarini for proofreading. CM was partially funded by a European Union FP7 grant (EMBALANCE: Grant Agreement no. 610454), the Brainlinks-Braintools Cluster of Excellence funded by the German Research foundation (DFG, grant no. ADV139), and by DFG (MA 2543/3-1).

normal people. J. Am. Geriatr. Soc. 42, 405-412. doi:10.1111/j.1532-5415.1994. tb07489.x

Bergström, B. (1973). Morphology of the vestibular nerve: II. the number of myelinated vestibular nerve fibers in man at various ages. Acta Otolaryngol. 76, 173-179. doi:10.3109/00016487309121496

Black, F. O. (2001). What can posturography tell us about vestibular function? Ann. N. Y. Acad. Sci. 942, 446-464. doi:10.1111/j.1749-6632. 2001.tb03765.x

Bohannon, R. (2006). Reference values for the timed up and go test: a descriptive meta-analysis. J. Geriatr. Phys. Ther. 29, 64-68. doi:10.1519/ 00139143-200608000-00004 
Buatois, S., Gueguen, R., Gauchard, G. C., Benetos, A., and Perrin, P. P. (2006). Posturography and risk of recurrent falls in healthy non-institutionalized persons aged over 65. Gerontology 52, 345-352. doi:10.1159/000094983

Cenciarini, M., Loughlin, P. J., Sparto, P. J., and Redfern, M. S. (2009). "Mediallateral postural control in older adults exhibits increased stiffness and damping," in Conference Proceedings: Annual International Conference of the IEEE Engineering in Medicine and Biology Society. Annual Conference, 2009 (Minneapolis, Minnesota: IEEE Engineering in Medicine and Biology Society), 7006-7009.

Cenciarini, M., Loughlin, P. J., Sparto, P. J., and Redfern, M. S. (2010). Stiffness and damping in postural control increase with age. IEEE Trans. Biomed. Eng. 57, 267-275. doi:10.1109/TBME.2009.2031874

Cnyrim, C., Mergner, T., and Maurer, C. (2009). Potential roles of force cues in human stance control. Exp. Brain Res. 194, 419-433. doi:10.1007/ s00221-009-1715-7

Collins, J. J., De Luca, C. J., Burrows, A., and Lipsitz, L. A. (1995). Age-related changes in open-loop and closed-loop postural control mechanisms. Exp. Brain Res. 104, 480-492. doi:10.1007/BF00231982

Davidson, B. S., Madigan, M. L., Southward, S. C., and Nussbaum, M. A. (2011). Neural control of posture during small magnitude perturbations: effects of aging and localized muscle fatigue. IEEE Trans. Biomed. Eng. 58, 1546-1554. doi:10.1109/TBME.2010.2095500

Delignières, D., Torre, K., and Bernard, P. (2011). Transition from persistent to antipersistent correlations in postural sway indicates velocity-based control. PLoS Comput. Biol. 7:e1001089. doi:10.1371/journal.pcbi.1001089

Doherty, T. J. (2003). Invited review: aging and sarcopenia. J. Appl. Physiol. 95, 1717-1727. doi:10.1152/japplphysiol.00347.2003

Duncan, P. W., Weiner, D. K., Chandler, J., and Studenski, S. (1990). Functional reach: a new clinical measure of balance. J. Gerontol. 45, M192-M197. doi:10. 1093/geronj/45.6.M192

Eikema, D. J., Hatzitaki, V., Tzovaras, D., and Papaxanthis, C. (2012). Age-dependent modulation of sensory reweighting for controlling posture in a dynamic virtual environment. Age 34, 1381-1392. doi:10.1007/s11357-011-9310-9

Eikema, D. J., Hatzitaki, V., Tzovaras, D., and Papaxanthis, C. (2014). Application of intermittent galvanic vestibular stimulation reveals age-related constraints in the multisensory reweighting of posture. Neurosci. Lett. 561, 112-117. doi:10. 1016/j.neulet.2013.12.048

Engelhart, D., Pasma, J. H., Schouten, A. C., Meskers, C. G. M., Maier, A. B., Mergner, T., et al. (2014). Impaired standing balance in elderly: a new engineering method helps to unravel causes and effects. J. Am. Med. Dir. Assoc. 15, .e1-.e6. doi:10.1016/j.jamda.2013.09.009

Enkelaar, L., Smulders, E., van Schrojenstein Lantman-de Valk, H., Weerdesteyn, V., and Geurts, A. C. H. (2013). Clinical measures are feasible and sensitive to assess balance and gait capacities in older persons with mild to moderate intellectual disabilities. Res. Dev. Disabil. 34, 276-285. doi:10.1016/j.ridd.2012.08.014

Era, P., and Heikkinen, E. (1985). Postural sway during standing and unexpected disturbance of balance in random samples of men of different ages. J. Gerontol. 40, 287-295. doi:10.1093/geronj/40.3.287

Fransson, P.-A., Kristinsdottir, E. K., Hafström, A., Magnusson, M., and Johansson, R. (2004). Balance control and adaptation during vibratory perturbations in middle-aged and elderly humans. Eur. J. Appl. Physiol. 91, 595-603. doi:10.1007/ s00421-003-1013-1

Ghulyan, V., Paolino, M., Lopez, C., Dumitrescu, M., and Lacour, M. (2005). A new translational platform for evaluating aging or pathology-related postural disorders. Acta Otolaryngol. 125, 607-617. doi:10.1080/00016480510026908

Goble, D. J., Coxon, J. P., Wenderoth, N., van Impe, A., and Swinnen, S. P. (2009). Proprioceptive sensibility in the elderly: degeneration, functional consequences and plastic-adaptive processes. Neurosci. Biobehav. Rev. 33, 271-278. doi:10. 1016/j.neubiorev.2008.08.012

Granacher, U., Gruber, M., and Gollhofer, A. (2009). Resistance training and neuromuscular performance in seniors. Int. J. Sports Med. 30, 652-657. doi:10. 1055/s-0029-1224178

Grossniklaus, H. E., Nickerson, J. M., Edelhauser, H. F., Bergman, L. A. M. K., and Berglin, L. (2013). Anatomic alterations in aging and age-related diseases of the eye. Invest. Ophthalmol. Vis. Sci. 54, ORSF23-ORSF27. doi:10.1167/iovs. 13-12711

Hilz, M. J., Axelrod, F. B., Hermann, K., Haertl, U., Duetsch, M., and Neundörfer, B. (1998). Normative values of vibratory perception in 530 children, juveniles and adults aged 3-79 years. J. Neurol. Sci. 159, 219-225. doi:10.1016/S0022-510X(98) 00177-4
Horak, F. B., Shupert, C. L., and Mirka, A. (1989). Components of postural dyscontrol in the elderly: a review. Neurobiol. Aging 10, 727-738. doi:10.1016/ 0197-4580(89)90010-9

Hytönen, M., Pyykkö, I., Aalto, H., and Starck, J. (1993). Postural control and age. Acta Otolaryngol. 113, 119-122. doi:10.3109/00016489309135778

Iosa, M., Fusco, A., Morone, G., and Paolucci, S. (2014). Development and decline of upright gait stability. Front. Aging Neurosci. 6:14. doi:10.3389/fnagi.2014.00014

Ishida, A., Imai, S., and Fukuoka, Y. (1997). Analysis of the posture control system under fixed and sway-referenced support conditions. IEEE Trans. Biomed. Eng. 44, 331-336. doi:10.1109/10.568908

Jeka, J., Allison, L., Saffer, M., Zhang, Y., Carver, S., and Kiemel, T. (2006). Sensory reweighting with translational visual stimuli in young and elderly adults: the role of state-dependent noise. Exp. Brain Res. 174, 517-527. doi:10.1007/ s00221-006-0502-y

Klass, M., Baudry, S., and Duchateau, J. (2007). Voluntary activation during maximal contraction with advancing age: a brief review. Eur. J. Appl. Physiol. 100, 543-551. doi:10.1007/s00421-006-0205-x

Kuo, A., Speers, R., Peterka, R., and Horak, F. (1998). Effect of altered sensory conditions on multivariate descriptors of human postural sway. Exp. Brain Res. 122, 185-195. doi:10.1007/s002210050506

Liaw, M.-Y., Chen, C.-L., Pei, Y.-C., Leong, C.-P., and Lau, Y.-C. (2009). Comparison of the static and dynamic balance performance in young, middle-aged, and elderly healthy people. Chang Gung Med. J. 32, 297-304.

Lin, Y.-H., Hsieh, S.-C., Chao, C.-C., Chang, Y.-C., and Hsieh, S.-T. (2005). Influence of aging on thermal and vibratory thresholds of quantitative sensory testing. $J$. Peripher. Nerv. Syst. 10, 269-281. doi:10.1111/j.1085-9489.2005.10305.x

Macaluso, A., and De Vito, G. (2004). Muscle strength, power and adaptations to resistance training in older people. Eur. J. Appl. Physiol. 91, 450-472. doi:10. 1007/s00421-003-0991-3

Maitre, J., Gasnier, Y., Bru, N., Jully, J.-L., and Paillard, T. (2013). Discrepancy in the involution of the different neural loops with age. Eur. J. Appl. Physiol. 113, 1821-1831. doi:10.1007/s00421-013-2608-9

Maki, B. E., Holliday, P. J., and Fernie, G. R. (1990). Aging and postural control. A comparison of spontaneous- and induced-sway balance tests. J. Am. Geriatr. Soc. 38, 1-9. doi:10.1111/j.1532-5415.1990.tb01588.x

Mansfield, A., and Maki, B. E. (2009). Are age-related impairments in change-insupport balance reactions dependent on the method of balance perturbation? J. Biomech. 42, 1023-1031. doi:10.1016/j.jbiomech.2009.02.007

Masani, K., Vette, A. H., and Popovic, M. R. (2006). Controlling balance during quiet standing: proportional and derivative controller generates preceding motor command to body sway position observed in experiments. Gait Posture 23, 164-172. doi:10.1016/j.gaitpost.2005.01.006

Maurer, C., Mergner, T., and Peterka, R. J. (2004). Abnormal resonance behavior of the postural control loop in Parkinson's disease. Exp. Brain Res. 157, 369-376. doi:10.1007/s00221-004-1852-y

Maurer, C., Mergner, T., and Peterka, R. J. (2006a). Multisensory control of human upright stance. Exp. Brain Res. 171, 231-250. doi:10.1007/s00221-005-0256-y

Maurer, C., Schweigart, G., and Mergner, T. (2006b). Pronounced overestimation of support surface tilt during stance. Exp. Brain Res. 168, 41-50. doi:10.1007/ s00221-005-0087-x

Maurer, C., Mergner, T., Xie, J., Faist, M., Pollak, P., and Lücking, C. H. (2003). Effect of chronic bilateral subthalamic nucleus (STN) stimulation on postural control in Parkinson's disease. Brain 126, 1146-1163. doi:10.1093/brain/awg100

Maurer, C., and Peterka, R. J. (2005). A new interpretation of spontaneous sway measures based on a simple model of human postural control. J. Neurophysiol. 93, 189-200. doi:10.1152/jn.00221.2004

Merchant, S. N., Velázquez-Villaseñor, L., Tsuji, K., Glynn, R. J., Wall, C III., and Rauch, S. D. (2000). Temporal bone studies of the human peripheral vestibular system. Normative vestibular hair cell data. Ann. Otol. Rhinol. Laryngol. Suppl. 181, 3-13.

Mergner, T., Maurer, C., and Peterka, R. J. (2002). Sensory contributions to the control of stance: a posture control model. Adv. Exp. Med. Biol. 508, 147-152. doi:10.1007/978-1-4615-0713-0_18

Mergner, T., Maurer, C., and Peterka, R. J. (2003). A multisensory posture control model of human upright stance. Prog. Brain Res. 142, 189-201. doi:10.1016/ S0079-6123(03)42014-1

Moghadam, M., Ashayeri, H., Salavati, M., Sarafzadeh, J., Taghipoor, K., Saeedi, A., et al. (2011). Reliability of center of pressure measures of postural stability in healthy older adults: effects of postural task difficulty and cognitive load. Gait Posture 33, 651-655. doi:10.1016/j.gaitpost.2011.02.016 
Nag, T. C., and Wadhwa, S. (2012). Ultrastructure of the human retina in aging and various pathological states. Micron 43, 759-781. doi:10.1016/j.micron.2012. 01.011

Nagy, E., Feher-Kiss, A., Barnai, M., Domján-Preszner, A., Angyan, L., and Horvath, G. (2007). Postural control in elderly subjects participating in balance training. Eur. J. Appl. Physiol. 100, 97-104. doi:10.1007/s00421-007-0407-x

Nashner, L. M., Black, F. O., and Wall, C. III (1982). Adaptation to altered support and visual conditions during stance: patients with vestibular deficits. J. Neurosci. 2, 536-544.

Nashner, L. M., and McCollum, G. (1985). The organization of human postural movements: a formal basis and experimental synthesis. Behav Brain Sci. 8, 135-150. doi:10.1017/S0140525X00020008

Nishihori, T., Aoki, M., Jiang, Y., Nagasaki, S., Furuta, Y., and Ito, Y. (2012). Effects of aging on lateral stability in quiet stance. Aging Clin. Exp. Res. 24, 162-170. doi: $10.3275 / 7626$

Papegaaij, S., Taube, W., Baudry, S., Otten, E., and Hortobágyi, T. (2014). Aging causes a reorganization of cortical and spinal control of posture. Front. Aging Neurosci. 6:28. doi:10.3389/fnagi.2014.00028

Park, J. J., Tang, Y., Lopez, I., and Ishiyama, A. (2001). Age-related change in the number of neurons in the human vestibular ganglion. J. Comp. Neurol. 431, 437-443. doi:10.1002/1096-9861(20010319)431:4<437::AID-CNE1081>3. $0 . \mathrm{CO} ; 2-\mathrm{P}$

Peterka, R. J. (2000). Postural control model interpretation of stabilogram diffusion analysis. Biol. Cybern. 82, 335-343. doi:10.1007/s004220050587

Peterka, R. J. (2002). Sensorimotor integration in human postural control. J. Neurophysiol. 88, 1097-1118. doi:10.1152/jn.00605.2001

Peterka, R. J., and Black, F. O. (1990). Age-related changes in human posture control: sensory organization tests. J. Vestib. Res. 1, 73-85.

Pierchała, K., Lachowska, M., Morawski, K., and Niemczyk, K. (2012). Sensory organization test outcomes in young, older and elderly healthy individuals preliminary results. Otolaryngol. Pol. 66, 274-279. doi:10.1016/j.otpol.2012.05. 005

Podsiadlo, D., and Richardson, S. (1991). The timed "Up \& Go": a test of basic functional mobility for frail elderly persons. J. Am. Geriatr. Soc. 39, 142-148. doi:10.1111/j.1532-5415.1991.tb01616.x

Prieto, T. E., Myklebust, J. B., Hoffmann, R. G., Lovett, E. G., and Myklebust, B. M. (1996). Measures of postural steadiness: differences between healthy young and elderly adults. IEEE Trans. Biomed. Eng. 43, 956-966. doi:10.1109/10.532130

Qu, X., Nussbaum, M. A., and Madigan, M. L. (2009). Model-based assessments of the effects of age and ankle fatigue on the control of upright posture in humans. Gait Posture 30, 518-522. doi:10.1016/j.gaitpost.2009.07.127

Rauch, S. D., Velázquez-Villaseñor, L., Dimitri, P. S., and Merchant, S. N. (2001). Decreasing hair cell counts in aging humans. Ann. N. Y. Acad. Sci. 942, 220-227. doi:10.1111/j.1749-6632.2001.tb03748.x

Reeves, N. D., Narici, M. V., and Maganaris, C. N. (2006). Myotendinous plasticity to ageing and resistance exercise in humans. Exp. Physiol. 91, 483-498. doi:10. 1113/expphysiol.2005.032896

Rosengren, K. S., Rajendran, K., Contakos, J., Chuang, L.-L., Peterson, M., Doyle, R., et al. (2007). Changing control strategies during standard assessment using computerized dynamic posturography with older women. Gait Posture 25, 215-221. doi:10.1016/j.gaitpost.2006.03.009

Rosenhall, U. (1973). Degenerative patterns in the aging human vestibular neuroepithelia. Acta Otolaryngol. 76, 208-220. doi:10.3109/00016487309121501

Ruhe, A., Fejer, R., and Walker, B. (2010). The test-retest reliability of centre of pressure measures in bipedal static task conditions - a systematic review of the literature. Gait Posture 32, 436-445. doi:10.1016/j.gaitpost.2010.09.012
Schieppati, M., Tacchini, E., Nardone, A., Tarantola, J., and Corna, S. (1999). Subjective perception of body sway. J. Neurol. Neurosurg. Psychiatr. 66, 313-322. doi:10.1136/jnnp.66.3.313

Shaffer, S. W., and Harrison, A. L. (2007). Aging of the somatosensory system: a translational perspective. Phys. Ther. 87, 193-207. doi:10.2522/ptj.20060083

Shumway-Cook, A., and Woollacott, M. (2001). "Chapter 9: aging and postural control," in Motor Control: Theory and Practical Applications, 2nd Edn, ed. M. Biblis (Philadelphia, PA: Lippincott Williams \& Wilkins), 222-247.

Speers, R. A., Kuo, A. D., and Horak, F. B. (2002). Contributions of altered sensation and feedback responses to changes in coordination of postural control due to aging. Gait Posture 16, 20-30. doi:10.1016/S0966-6362(02)00003-6

Tang, P., and Woollacott, M. (1996). "Balance control in the elderly," in Clinical Disorders of Balance, Posture and Gait, eds A. Bronstein, T. Brandt, and M. Woollacott (London: Arnold), 268-286.

Teasdale, N., and Simoneau, M. (2001). Attentional demands for postural control: the effects of aging and sensory reintegration. Gait Posture 14, 203-210. doi:10. 1016/S0966-6362(01)00134-5

Teasdale, N., Stelmach, G. E., and Breunig, A. (1991). Postural sway characteristics of the elderly under normal and altered visual and support surface conditions. $J$. Gerontol. 46, B238-B244. doi:10.1093/geronj/46.6.B238

Tia, B., Paizis, C., Mourey, F., and Pozzo, T. (2012). Do equilibrium constraints modulate postural reaction when viewing imbalance? Brain Cogn. 79, 89-95 doi:10.1016/j.bandc.2012.02.008

Tsai, Y.-C., Hsieh, L.-F., and Yang, S. (2014). Age-related changes in posture response under a continuous and unexpected perturbation. J. Biomech. 47, 482-490. doi:10.1016/j.jbiomech.2013.10.047

Van der Kooij, H., Jacobs, R., Koopman, B., and van der Helm, F. (2001). An adaptive model of sensory integration in a dynamic environment applied to human stance control. Biol. Cybern. 84, 103-115. doi:10.1007/s004220000196

Van der Kooij, H., and Peterka, R. J. (2011). Non-linear stimulus-response behavior of the human stance control system is predicted by optimization of a system with sensory and motor noise. J. Comput. Neurosci. 30, 759-778. doi:10.1007/ s10827-010-0291-y

Vette, A. H., Masani, K., Nakazawa, K., and Popovic, M. R. (2010). Neuralmechanical feedback control scheme generates physiological ankle torque fluctuation during quiet stance. IEEE Trans. Neural. Syst. Rehabil. Eng. 18, 86-95 doi:10.1109/TNSRE.2009.2037891

Whipple, R., Wolfson, L., Derby, C., Singh, D., and Tobin, J. (1993). Altered sensory function and balance in older persons. J. Gerontol. 48, 71-76. doi:10.1093/ geronj/48.Special_Issue.71

Winter, D. (1995). Human balance and posture control during standing and walking. Gait Posture 3, 193-214. doi:10.1016/0966-6362(95)99069-W

Woollacott, M., Inglin, B., and Manchester, D. (1988). Response preparation and posture control neuromuscular changes in the older adult. Ann. N. Y. Acad. Sci. 515, 42-53. doi:10.1111/j.1749-6632.1988.tb32964.x

Woollacott, M. H., and Shumway-Cook, A. (1990). Changes in posture control across the life span - a systems approach. Phys. Ther. 70, 799-807.

Conflict of Interest Statement: The authors declare that the research was conducted in the absence of any commercial or financial relationships that could be construed as a potential conflict of interest.

Copyright (c) 2015 Wiesmeier, Dalin and Maurer. This is an open-access article distributed under the terms of the Creative Commons Attribution License (CC BY). The use, distribution or reproduction in other forums is permitted, provided the original author(s) or licensor are credited and that the original publication in this journal is cited, in accordance with accepted academic practice. No use, distribution or reproduction is permitted which does not comply with these terms. 